\title{
A Reconstruction Procedure Associated with Switching Lyapunov Function for Relaxing Stability Assurance of T-S Fuzzy Mode
}

\author{
Yau-Tarng Juang, ${ }^{1}$ Chih-Peng Huang, ${ }^{2}$ and Chung-Lin Yan ${ }^{1}$ \\ ${ }^{1}$ Department of Electrical Engineering, National Central University, Jhongli City, Taoyuan County 320, Taiwan \\ ${ }^{2}$ Department of Computer Science, University of Taipei, Taipei 100, Taiwan \\ Correspondence should be addressed to Chih-Peng Huang; ponytony@seed.net.tw
}

Received 1 August 2014; Accepted 27 September 2014

Academic Editor: Junuthula N. Reddy

Copyright (C) 2015 Yau-Tarng Juang et al. This is an open access article distributed under the Creative Commons Attribution License, which permits unrestricted use, distribution, and reproduction in any medium, provided the original work is properly cited.

\begin{abstract}
This paper proposes a novel reconstruction procedure to lessen the conservatism of stability assurance of T-S Fuzzy Mode. By dividing the state variables into some bounded regions, the considered T-S fuzzy model can be first transferred to an alternative form via a reconstructing procedure. Thus, we can attain some relaxing stability criteria based on the switching quadratic Lyapunov function (SQLF) method. Notably, these proposed conditions are explicitly formulated by linear matrix inequality (LMI) form and can handily be evaluated by current software tools. Finally some illustrative examples are given to experimentally demonstrate the validity and merit of the proposed method.
\end{abstract}

\section{Introduction}

A class of empirical control architecture, fuzzy logic control (FLC), is originally proposed in the past two decades [1]. And, it then has become one of the most active and fruitful areas in this research community. Many researchers have dedicated a lot of time and great effort to both theoretical research and implementation techniques for FLC. The basic idea behind FLC is to incorporate the "expert experience" of a human operator into a system. Notably, a complicated dynamic model is not necessary any more. However, modelfree type of fuzzy control is useful and practical, but this control technique is insufficient of mathematical support. In order to systematically analyze the stability of the fuzzy system, Takagi-Sugeno (T-S) fuzzy model [2,3] was presented to open a different viewpoint from the traditional fuzzy system. Based on the T-S fuzzy model, a complicated system can be represented as a set of fuzzy IF-THEN rules, each of which represents the local linear subsystem in a different state-space region. By the fuzzy blending operating, they thus can approximately represent some uncertain or/and nonlinear systems [4-8].
The stability issues of T-S fuzzy model were first analyzed by Tanaka and Sugeno [3]. Based on Lyapunov direct method, they presented some sufficient conditions that could systematically achieve the stability analysis and stabilization of fuzzy systems $[9,10]$. Moreover, the stability conditions could be cast into linear matrix inequality (LMI) forms $[1,10$ 13]. For earlier research, most works are devoted to common quadratic Lyapunov function (CQLF) methods. Thus, the proposed criteria require finding a common positive definite matrix $\mathbf{P}$ of a CQLF to satisfy all the subsystems [3, 14-19]. But, if the number of fuzzy rules is large, a common matrix $\mathbf{P}$ may hardly be obtained or does not exist in all the subsystems. Instead of CQLF, a switching quadratic Lyapunov function (SQLF) was involved [20-24]. This SQLF method beforehand divides the state space into several regions, and, in each region we can try to find the individual positive-definite matrix $\mathbf{P}_{i}$ for the SQLF. Furthermore, we need to guarantee the SQLF $V_{i}(\mathbf{x}(t))$ be the same in the region boundary but did not expect that all $\mathbf{P}_{i}$ are equal. In previous works, the stability criteria need to involve bilinear matrix inequality (BMI) conditions, which cannot be directly evaluated by traditional LMI solver [25]. 
In this work, based on SQLF method, we devote the stability criteria of T-S fuzzy model to the relaxing. Firstly, we use a distinct region-division approach for the state space. When assuming that the state variables of the considered T-S fuzzy model can be correspondingly formulated by single membership function at the region boundary, we thus propose a reconstructing procedure for substituting. Thus, based on the SQLF method, more relaxed stability criteria can be derived and expressed by LMI manner. Finally, an illustrative example is given to verify the efficiency and superiority of the proposed method.

\section{Problem Description and Preliminaries}

T-S fuzzy model can approximately represent some intractable systems with parametric uncertainty or nonlinearity via the fuzzy IF-THEN rules interpretation. This mode mainly characterizes the local dynamics behaviors of a system by the fuzzy rules where their consequent parts are represented by some locally linear models. Thus, an overall system can be achieved via the fuzzy "blending" for these linear models. The $r$ rules of a T-S fuzzy model are expressed as follows:

$$
\begin{aligned}
& \text { Model Rule } i-\left(\mathrm{MR}_{i}\right) \text { : } \\
& \text { IF } x_{1}(t) \text { is } M_{i 1} \text { and } x_{2}(t) \text { is } M_{i 2} \cdots \text { and } x_{n}(t) \text { is } M_{i n} \text {, } \\
& \text { THEN } \dot{\mathbf{x}}(t)=\mathbf{A}_{i} \mathbf{x}(t) \quad i=1,2, \ldots, r,
\end{aligned}
$$

where $\mathrm{MR}_{i}$ denotes the model rule $i ; M_{i j}$ denotes the fuzzy set corresponding to the state variable $x_{j}(t)$ of $\mathrm{MR}_{i}$; $r$ is the number of IF-THEN fuzzy model rules; $\mathbf{x}(t)=$ $\left[\begin{array}{llll}x_{1}(t) & x_{2}(t) & \cdots & x_{n}(t)\end{array}\right]^{T} \in \mathfrak{R}^{n}$ is the state vector; $\mathbf{A}_{i} \in$ $\mathfrak{R}^{n \times n}$ denotes the system matrix of the subsystem.

The T-S fuzzy model in (1) is assumed to be inferred by center average defuzzification (CAD) and can be described by

$$
\dot{\mathbf{x}}(t)=\frac{\sum_{i=1}^{r} w_{i}(\mathbf{x}(t)) \mathbf{A}_{i} \mathbf{x}(t)}{\sum_{i=1}^{r} w_{i}(\mathbf{x}(t))}=\sum_{i=1}^{r} h_{i}(\mathbf{x}(t)) \mathbf{A}_{i} \mathbf{x}(t),
$$

where

$$
\begin{array}{r}
w_{i}(\mathbf{x}(t))=\prod_{j=1}^{n} M_{i j}\left(x_{j}(t)\right), \\
\sum_{i=1}^{r} w_{i}(\mathbf{x}(t))>0, \quad w_{i}(\mathbf{x}(t)) \geq 0, \\
i=1,2, \ldots, r .
\end{array}
$$

$M_{i j}\left(x_{j}(t)\right)$ is the grade of membership of $x_{j}(t)$ in membership function $M_{i j}$. And, the inferred grade function is normalized as

$$
\begin{gathered}
h_{i}(\mathbf{x}(t))=\frac{w_{i}(\mathbf{x}(t))}{\sum_{i=1}^{r} w_{i}(\mathbf{x}(t))}, \\
\sum_{i=1}^{r} h_{i}(\mathbf{x}(t))=1, \quad h_{i}(\mathbf{x}(t)) \geq 0, \\
i=1,2, \ldots, r .
\end{gathered}
$$

Based on Lyapunov stability theory, a candidate Lyapunov function with a constant matrix $P$ is given by

$$
V(\mathbf{x}(t))=\mathbf{x}^{T}(t) \mathbf{P x}(t)
$$

with $P>0$. Thus, for stability assurance, the chosen Lyapunov function needs to satisfy the following four conditions:

$$
\begin{aligned}
& \text { (1) } V \text { is } C^{1}, \\
& \text { (2) } \mathbf{x}(t)=\mathbf{0} \Leftrightarrow V(\mathbf{x}(t))=0, \\
& \text { (3) } \mathbf{x}(t) \neq \mathbf{0} \Leftrightarrow V(\mathbf{x}(t))>0, \\
& \text { (4) }\|\mathbf{x}(t)\| \rightarrow \infty \Rightarrow V(\mathbf{x}(t)) \rightarrow \infty,
\end{aligned}
$$

where $C^{1}$ is the set of all real-valued functions that have a continuous first derivative on $(-\infty, \infty)$ and $\|\cdot\|$ denotes the Euclidean norm for the considered vectors.

Based on (5), Tanaka and Sugeno [3] originally proposed a stability criterion as follows.

Lemma 1 (see [3]). The equilibrium of the autonomous T-S fuzzy system in (2) is asymptotically stable in the large, if there exists a common positive-definite symmetric matrix $P$ such that

$$
\mathbf{A}_{i}^{T} \mathbf{P}+\mathbf{P A}_{i}<\mathbf{0}, \quad i=1,2, \ldots, r .
$$

Remark 2. Lemma 1 shows that we need to find a common matrix $P$ to satisfy all the conditions in (6) for global stability assurance. Based on Lemma 1, some less conservative results $[12,16]$ with a common positive-definite symmetric matrix $P$ were achieved by involving some extra slack matrices in criteria.

\section{Switching T-S Fuzzy Model and Stability Issues}

\subsection{Switching T-S Fuzzy Model. Consider}

Region Model Rule $q-\left(\mathrm{RMR}_{q}\right)$ :

IF $\mathbf{x}(t) \in$ Region $q$

THEN

Local Model Rule $i-\left(\mathrm{LMR}_{i}\right)$ :

IF $x_{1}(t)$ is $M_{q i 1}$ and $x_{2}(t)$ is $M_{q i 2} \cdots$ and $x_{n}(t)$ is $M_{q i n}$,

$\operatorname{THEN} \dot{\mathbf{x}}(t)=\mathbf{A}_{q i} \mathbf{x}(t)+\mathbf{B}_{q i} \mathbf{u}(t)$

$$
i=1,2, \ldots, r_{q}, \quad q=1,2, \ldots, 2^{n}
$$

where $\mathrm{RMR}_{q}$ denotes the region model rule $q . \mathrm{LMR}_{i}$ denotes the local model rule $i . M_{q i j}$ denotes the fuzzy set corresponding to the state variable $x_{j}(t)$ of $\mathrm{LMR}_{i}$ in Region $q . r_{q}$ is the number of IF-THEN fuzzy local model rules in Region $q .2^{n}$ is the number of region. $n$ is the number of state variables. $r=\sum_{q=1}^{2^{n}} r_{q}$ is the total number of IF-THEN fuzzy model rules. $\mathbf{A}_{q i} \in \mathfrak{R}^{n \times n}$ denotes the system matrix of the subsystem. $\mathbf{B}_{q i} \in \mathfrak{R}^{n \times m}$ denotes the input matrix of the subsystem. 


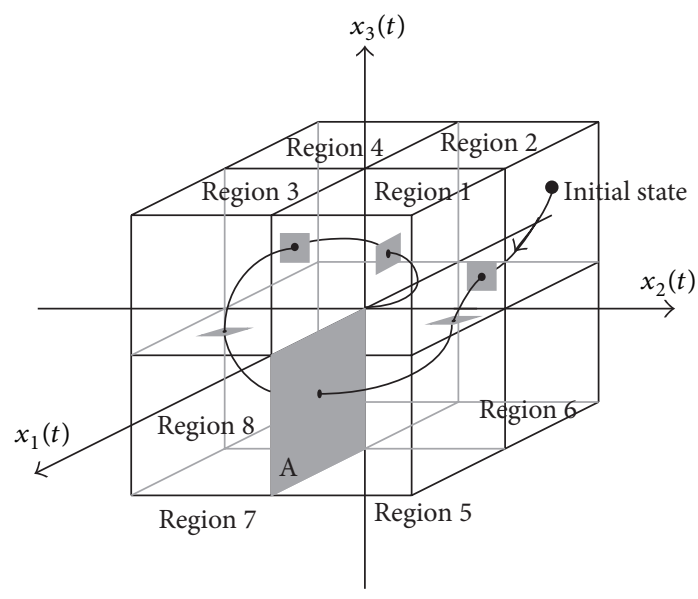

A plane on region boundary

FIGURE 1: Divided state space and change of state.

Each linear consequent equation, $\mathbf{A}_{q i} \mathbf{x}(t)+\mathbf{B}_{q i} \mathbf{u}(t)$, is called a subsystem.

Given a pair of $(\mathbf{x}(t), \mathbf{u}(t))$, the final output of the T-S fuzzy model (7) is inferred by CAD and can be represented as follows [23]:

$$
\dot{\mathbf{x}}(t)=\sum_{q=1}^{2^{n}} \sum_{i=1}^{r_{q}} \eta_{q}(\mathbf{x}(t)) h_{q i}(\mathbf{x}(t))\left\{\mathbf{A}_{q i} \mathbf{x}(t)+\mathbf{B}_{q i} \mathbf{u}(t)\right\},
$$

where

$$
\eta_{q}(\mathbf{x}(t))= \begin{cases}1, & \mathbf{x}(t) \in R_{q} \\ 0, & \mathbf{x}(t) \notin R_{q}\end{cases}
$$

$h_{q i}(\mathbf{x}(t))$ is the normalized membership function of the $\mathrm{LMR}_{i}$ in Region $q$ and satisfies

$$
\begin{array}{r}
\sum_{q=1}^{2^{n}} \sum_{i=1}^{r_{q}} \eta_{q}(\mathbf{x}(t)) h_{q i}(\mathbf{x}(t))=1, \\
\sum_{q=1}^{2^{n}} \sum_{i=1}^{r_{q}} \eta_{q}(\mathbf{x}(t)) h_{q i}(\mathbf{x}(t)) \geq 0, \\
i=1,2, \ldots, r_{q} .
\end{array}
$$

In the qth region, we define

$$
\begin{gathered}
R_{q}\left(s_{1 q}, s_{2 q}, \ldots, s_{n q}\right) \\
s_{k q}=\left\{\begin{array}{ll}
1, & x_{k}(t) \geq 0 \\
0, & x_{k}(t)<0
\end{array} \quad k=1,2, \ldots, n,\right.
\end{gathered}
$$

where the number of the state variables is equal to $n$ and its corresponding state space can be divided into $2^{n}$ region. For clarification, assume that the considered state space has three dimensions and each separated region corresponds to a quadrant. Figure 1 depicts that the trajectory of a given initial state passes over the different quadrants in a three-dimensional space. The state in Figure 1 passes through Region 2, Region 1, Region 5, Region 7, Region 3, Region 4, and Region 2 in turn. To guarantee the stability of T-S fuzzy system, we suppose that the trajectory of a system cannot be motionless on the region boundaries.

\subsection{Switching Quadratic Lyapunov Function. Consider}

$$
V(\mathbf{x}(t))= \begin{cases}\mathbf{x}^{T}(t) \mathbf{P}_{1} \mathbf{x}(t), & \mathbf{x}(t) \in \text { Region } 1 \\ \mathbf{x}^{T}(t) \mathbf{P}_{2} \mathbf{x}(t), & \mathbf{x}(t) \in \text { Region } 2 \\ \vdots & \vdots \\ \mathbf{x}^{T}(t) \mathbf{P}_{2^{n}} \mathbf{x}(t), & \mathbf{x}(t) \in \text { Region } 2^{n}\end{cases}
$$

where $\mathbf{P}_{q} \in \mathfrak{R}^{n \times n} \forall q$ are a set of positive-definite symmetric matrices. This function is continuous on region boundaries if $\mathbf{P}_{q}$ satisfies the following constraint [23]:

$$
\begin{gathered}
\mathbf{P}_{q}=\left[\begin{array}{cccc}
p_{11 \zeta(1, q)} & p_{12 \psi(1,2, q)} & \cdots & p_{1 n \psi(1, n, q)} \\
p_{12 \psi(1,2, q)} & p_{22 \zeta(2, q)} & \cdots & p_{2 n \psi(2, n, q)} \\
\vdots & \vdots & \ddots & \vdots \\
p_{1 n \psi(1, n, q)} & p_{2 n \psi(2, n, q)} & \cdots & p_{n n \zeta(n, q)}
\end{array}\right], \\
\zeta(k, q)=2-s_{k q}, \\
\psi(k, l, q)=-s_{k q}-2 s_{l q}+4 .
\end{gathered}
$$

Since the involved property is crucial for discussing the SQLF, an example is illustrated as follows.

Example 3 (see [23]). As in Figure 1, consider a system with three state variables $x_{1}(t), x_{2}(t)$, and $x_{3}(t)$. The state space can be divided into the following eight regions, eight quadrants:

$$
\begin{array}{ccc}
R_{1}(1,1,1), & R_{2}(0,1,1), & R_{3}(1,0,1), \\
R_{4}(0,0,1) ; & R_{5}(1,1,0), & R_{6}(0,1,0), \\
R_{7}(1,0,0), & R_{8}(0,0,0) .
\end{array}
$$

Based on the notation of a region, that is, $R_{q}\left(s_{1 q}, s_{2 q}, \ldots, s_{n q}\right)$, each $\mathbf{P}_{q}$ is represented as follows:

$$
\begin{array}{ll}
P_{1}=\left[\begin{array}{lll}
p_{111} & p_{121} & p_{131} \\
p_{121} & p_{221} & p_{231} \\
p_{131} & p_{231} & p_{331}
\end{array}\right], & P_{2}=\left[\begin{array}{lll}
p_{112} & p_{122} & p_{132} \\
p_{122} & p_{221} & p_{231} \\
p_{132} & p_{231} & p_{331}
\end{array}\right], \\
P_{3}=\left[\begin{array}{lll}
p_{111} & p_{123} & p_{131} \\
p_{123} & p_{222} & p_{232} \\
p_{131} & p_{232} & p_{331}
\end{array}\right], & P_{4}=\left[\begin{array}{lll}
p_{112} & p_{124} & p_{132} \\
p_{124} & p_{222} & p_{232} \\
p_{132} & p_{232} & p_{331}
\end{array}\right], \\
P_{5}=\left[\begin{array}{lll}
p_{111} & p_{121} & p_{133} \\
p_{121} & p_{221} & p_{233} \\
p_{133} & p_{233} & p_{332}
\end{array}\right], & P_{6}=\left[\begin{array}{lll}
p_{112} & p_{122} & p_{134} \\
p_{122} & p_{221} & p_{233} \\
p_{134} & p_{233} & p_{332}
\end{array}\right], \\
P_{7}=\left[\begin{array}{lll}
p_{111} & p_{123} & p_{133} \\
p_{123} & p_{222} & p_{234} \\
p_{133} & p_{234} & p_{332}
\end{array}\right], & P_{8}=\left[\begin{array}{lll}
p_{112} & p_{124} & p_{134} \\
p_{124} & p_{222} & p_{234} \\
p_{134} & p_{234} & p_{332}
\end{array}\right],
\end{array}
$$




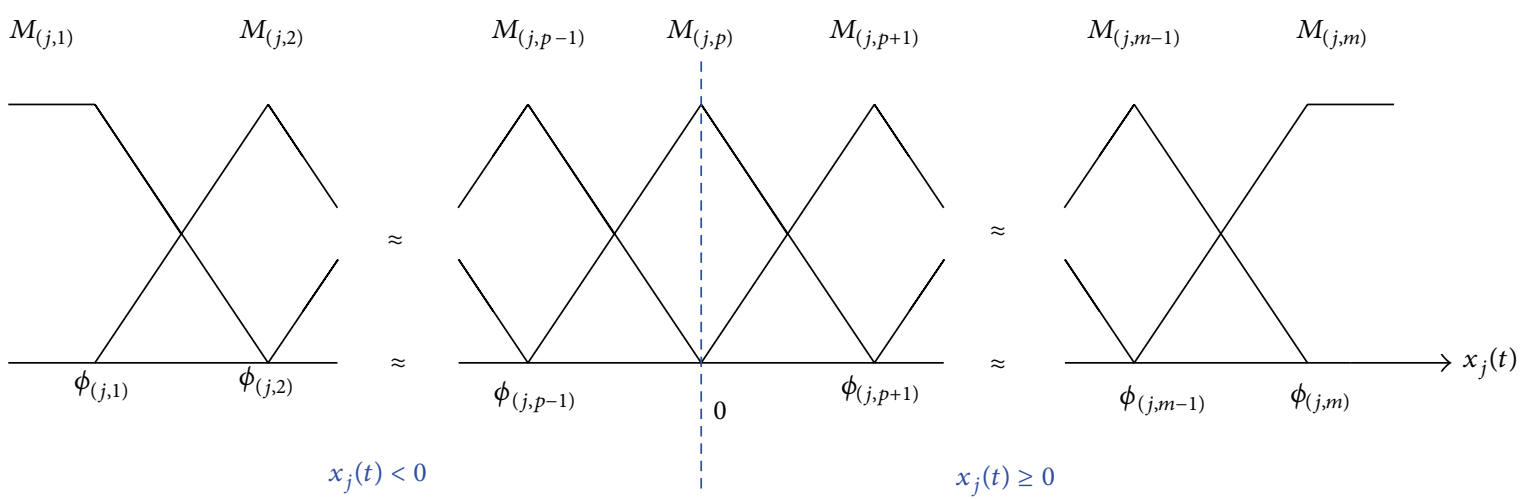

FIGURE 2: State variable $x_{j}(t)$ triggers one fuzzy set at $x_{j}(t)=0$.

On the region boundary between Regions 5 and Regions 7, a plane A in Figure 1 for $\mathbf{x}(t)=\left[\begin{array}{lll}x_{1}(t) & 0 & x_{3}(t)\end{array}\right]^{T}$, the SQLF is calculated as

$$
\begin{aligned}
V(\mathbf{x}(t)) & =\mathbf{x}^{T}(t) P_{5} \mathbf{x}(t) \\
& =\left[\begin{array}{lll}
x_{1}(t) & 0 & x_{3}(t)
\end{array}\right]\left[\begin{array}{lll}
p_{111} & p_{121} & p_{133} \\
p_{121} & p_{221} & p_{233} \\
p_{133} & p_{233} & p_{332}
\end{array}\right]\left[\begin{array}{c}
x_{1}(t) \\
0 \\
x_{3}(t)
\end{array}\right] \\
& =p_{111} x_{1}^{2}(t)+2 p_{133} x_{1}(t) x_{3}(t)+p_{332} x_{3}^{2}(t) \\
& =\left[\begin{array}{lll}
x_{1}(t) & 0 & x_{3}(t)
\end{array}\right]\left[\begin{array}{lll}
p_{111} & p_{123} & p_{133} \\
p_{123} & p_{222} & p_{234} \\
p_{133} & p_{234} & p_{332}
\end{array}\right]\left[\begin{array}{c}
x_{1}(t) \\
0 \\
x_{3}(t)
\end{array}\right] \\
& =\mathbf{x}^{T}(t) P_{7} \mathbf{x}(t) .
\end{aligned}
$$

Furthermore, the illustrative results can be similarly extended to the other region boundaries of $\mathbf{x}(t)$.

Remark 4. Based on the SQLF, (13), we have shown that the state trajectory crossed a plane; that is, $\mathbf{x}(t)=$ $\left[\begin{array}{lll}x_{1}(t) & 0 & x_{3}(t)\end{array}\right]^{T}$ is continuous. In addition, it is also continuous when the trajectory crosses a line, such as $\mathbf{x}(t)=$ $\left[\begin{array}{lll}0 & x_{2}(t) & 0\end{array}\right]^{T}[23]$.

\section{Main Result}

Considering in general fuzzy inference rules, a single state variable triggers no more than two fuzzy sets. Thus, fuzzy sets for a premise variable $x_{j}(t)$ can be assumed to have two different patterns, defined in Figures 2 and 3, respectively, where their differences are at $x_{j}(t)=0$ including single membership function or two membership functions.

For generalization and unification, our proposed approach merely focuses on addressing the pattern with $x_{j}(t)=0$ with single fuzzy set, that is, as in Figure 2. Thus, if the pattern is like Figure 3, we need to beforehand perform a reconstruction procedure described in the following.
By illustrating from the pattern in Figure 3, the reconstruction procedure has two stages. Stage 1 is to delete the two $M_{(j, p)}, M_{(j, p+1)}$ regarding $x_{j}(t)=0$ and retain the other fuzzy sets. Stage 2 is to establish three new fuzzy sets $M_{(j, p)}^{\prime}$, $M_{(j, 0)}$, and $M_{(j, p+1)}^{\prime}$ for $x_{j}(t)=0$ include single membership function. The achieved pattern can thus be depicted by Figure 4. The membership function $M_{(j, p)}^{\prime}\left(x_{j}(t)\right), M_{(j, 0)}\left(x_{j}(t)\right)$, and $M_{(j, p+1)}^{\prime}\left(x_{j}(t)\right)$ can be, respectively, constructed by

$$
\begin{aligned}
& M_{(j, p)}^{\prime}\left(x_{j}(t)\right) \\
& =\left\{\begin{array}{l}
M_{(j, p)}\left(x_{j}(t)\right), \\
\text { for } \phi_{(j, p-1)} \leq x_{j}(t) \leq \phi_{(j, p)} \\
M_{(j, p)}\left(x_{j}(t)\right)-\frac{M_{(j, p)}(0) M_{(j, p+1)}\left(x_{j}(t)\right)}{M_{(j, p+1)}(0)} \\
\text { for } \phi_{(j, p)} \leq x_{j}(t) \leq 0
\end{array}\right.
\end{aligned}
$$

$M_{(j, 0)}\left(x_{j}(t)\right)$

$= \begin{cases}\frac{M_{(j, p+1)}\left(x_{j}(t)\right)}{M_{(j, p+1)}(0)}, & \text { for } \phi_{(j, p)} \leq x_{j}(t) \leq 0 \\ \frac{M_{(j, p)}\left(x_{j}(t)\right)}{M_{(j, p)}(0)}, & \text { for } 0 \leq x_{j}(t) \leq \phi_{(j, p+1)}\end{cases}$

$$
M_{(j, p+1)}^{\prime}\left(x_{j}(t)\right)
$$

$$
= \begin{cases}M_{(j, p+1)}\left(x_{j}(t)\right)-\frac{M_{(j, p+1)}(0) M_{(j, p)}\left(x_{j}(t)\right)}{M_{(j, p)}(0)}, \\ M_{(j, p+1)}\left(x_{j}(t)\right), & \text { for } 0 \leq x_{j}(t) \leq \phi_{(j, p+1)} \\ & \text { for } \phi_{(j, p+1)} \leq x_{j}(t) \leq \phi_{(j, p+2)} .\end{cases}
$$




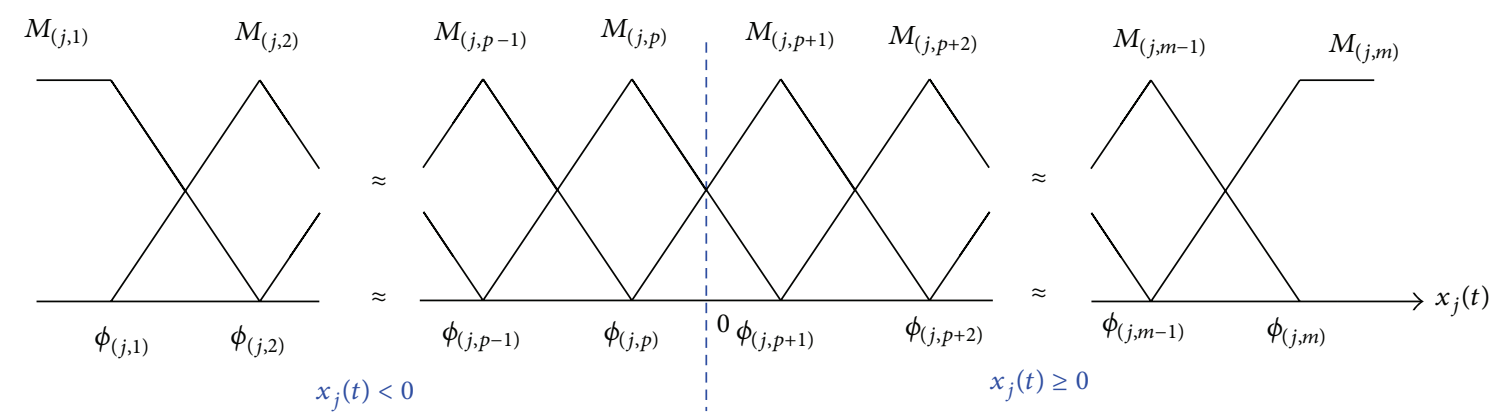

FIGURE 3: State variable $x_{j}(t)$ triggers two fuzzy set at $x_{j}(t)=0$.
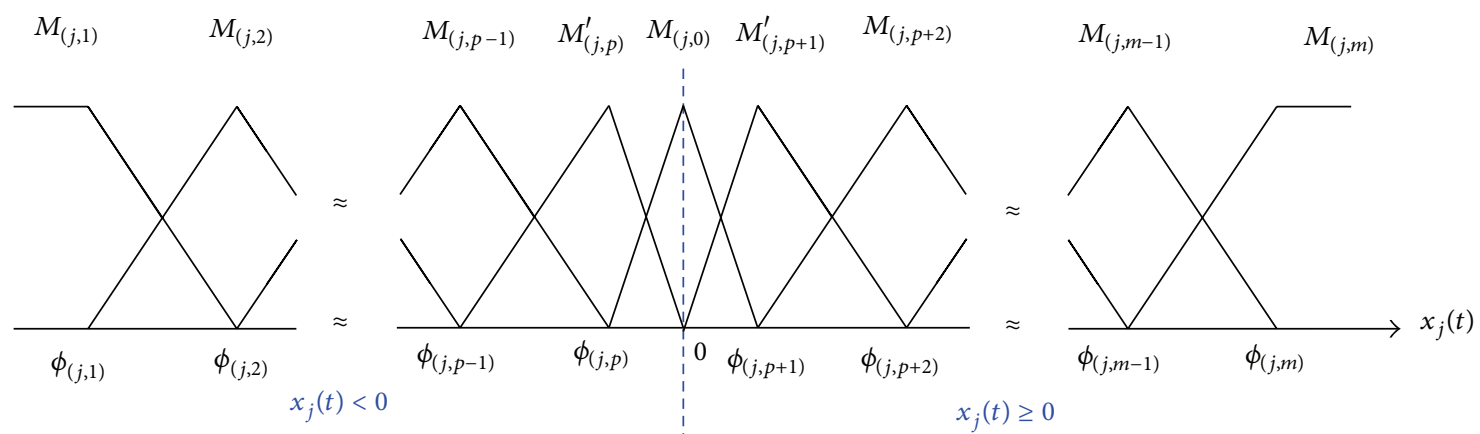

Figure 4: Reconstruction of fuzzy sets with state variable $x_{j}(t)$.

4.1. Reconstruction of Switching Takagi-Sugeno Fuzzy Model via Vertex Expression. Consider

$$
\begin{aligned}
& \text { Region Model Rule }\left(q_{1}, q_{2}, \ldots, q_{n}\right)-\left(\operatorname{RMR}_{\left(q_{1}, q_{2}, \ldots, q_{n}\right)}\right) \text { : } \\
& \text { IF } x_{1}(t) \text { is } N_{\left(1, q_{1}\right)} \text { and } x_{2}(t) \text { is } N_{\left(2, q_{2}\right)} \ldots \\
& \text { and } x_{n}(t) \text { is } N_{\left(n, q_{n}\right)},
\end{aligned}
$$

THEN

Local Model Rule $\left(i_{1}, i_{2}, \ldots, i_{n}\right)-\left(\operatorname{LMR}_{\left(i_{1}, i_{2}, \ldots, i_{n}\right)}\right):$

IF $x_{1}(t)$ is $M_{q_{1},\left(1, i_{1}\right)}$ and $x_{2}(t)$ is $M_{q_{2},\left(2, i_{2}\right)} \cdots$ and $x_{n}(t)$ is $M_{q_{n},\left(n, i_{n}\right)}$,

$$
\begin{aligned}
& \operatorname{THEN~} \dot{\mathbf{x}}(t)= \mathbf{A}_{\left(q_{1}, q_{2}, \ldots, q_{n}\right)\left(i_{1}, i_{2}, \ldots, i_{n}\right)} \mathbf{x}(t) \\
&+\mathbf{B}_{\left(q_{1}, q_{2}, \ldots, q_{n}\right)\left(i_{1}, i_{2}, \ldots, i_{n}\right)} \mathbf{u}(t) \\
& q_{j}=0,1, \quad i_{j}=1,2, \ldots, r_{j q_{j}}, \quad j=1,2, \ldots, n,
\end{aligned}
$$

where $\operatorname{RMR}_{\left(q_{1}, q_{2}, \ldots, q_{n}\right)}$ denotes the region model rule $\left(q_{1}\right.$, $\left.q_{2}, \ldots, q_{n}\right), \operatorname{LMR}_{\left(i_{1}, i_{2}, \ldots, i_{n}\right)}$ denotes the local model rule $\left(i_{1}, i_{2}\right.$, $\left.\ldots, i_{n}\right)$, and $N_{\left(j, q_{j}\right)}$ denotes the crisp set corresponding to the state variable $x_{j}(t)$ in divisional region $q_{j}$.
The membership function $N_{\left(j, q_{j}\right)}\left(x_{j}(t)\right)$ of crisp set $N_{\left(j, q_{j}\right)}$ is defined as below:

$$
\begin{aligned}
& N_{(j, 0)}\left(x_{j}(t)\right)= \begin{cases}1 & \text { for } x_{j}(t)<0 \\
0 & \text { for } x_{j}(t) \geq 0\end{cases} \\
& N_{(j, 1)}\left(x_{j}(t)\right)= \begin{cases}1 & \text { for } x_{j}(t) \geq 0 \\
0 & \text { for } x_{j}(t)<0 .\end{cases}
\end{aligned}
$$

$M_{q_{j},\left(j, i_{j}\right)}$ denotes the fuzzy set corresponding to the state variable $x_{j}(t)$ of $\operatorname{LMR}_{\left(i_{1}, i_{2}, \ldots, i_{n}\right)}$ in divisional region $q_{j} \cdot r_{j q_{j}}$ is the number of $x_{j}(t)$ 's fuzzy sets in divisional region $q_{j}$. $r_{\left(q_{1}, q_{2}, \ldots, q_{n}\right)}=\prod_{j=1}^{n} r_{j q_{j}}$ is the number of IF-THEN region model rule $\left(q_{1}, q_{2}, \ldots, q_{n}\right) r=\sum_{q_{1}=0}^{1} \sum_{q_{2}=0}^{1} \cdots \sum_{q_{n}=0}^{1} r_{\left(q_{1}, q_{2}, \ldots, q_{n}\right)}$ is the total number of IF-THEN fuzzy model rules. $\mathbf{A}_{\left(q_{1}, q_{2}, \ldots, q_{n}\right)\left(i_{1}, i_{2}, \ldots, i_{n}\right)} \in \mathfrak{R}^{n \times n}$ denotes the system matrix of the subsystem. And, $\mathbf{B}_{\left(q_{1}, q_{2}, \ldots, q_{n}\right)\left(i_{1}, i_{2}, \ldots, i_{n}\right)} \in \mathfrak{R}^{n \times m}$ denotes the input matrix of the subsystem.

Each linear consequent equation $\mathbf{A}_{\left(q_{1}, q_{2}, \ldots, q_{n}\right)\left(i_{1}, i_{2}, \ldots, i_{n}\right)} \mathbf{x}(t)+$ $\mathbf{B}_{\left(q_{1}, q_{2}, \ldots, q_{n}\right)\left(i_{1}, i_{2}, \ldots, i_{n}\right)} \mathbf{u}(t)$ is called a subsystem. Given a pair of $(\mathbf{x}(t), \mathbf{u}(t))$, the final output of the T-S fuzzy model (20) inferred by CAD can be represented as follows:

$\dot{\mathbf{x}}(t)=\sum_{q_{1}=0}^{1} \sum_{q_{2}=0}^{1} \ldots \sum_{q_{n}=0}^{1} \sum_{i_{1}=1}^{r_{1 q_{1}}} \sum_{i_{2}=1}^{r_{2 q_{2}}} \ldots \sum_{i_{n}=1}^{r_{n q_{n}}} \eta_{\left(q_{1}, q_{2}, \ldots, q_{n}\right)}(\mathbf{x}(t))$ 


$$
\begin{aligned}
& \times h_{\left(q_{1}, q_{2}, \ldots, q_{n}\right)\left(i_{1}, i_{2}, \ldots, i_{n}\right)}(\mathbf{x}(t)) \\
& \times\left\{\mathbf{A}_{\left(q_{1}, q_{2}, \ldots, q_{n}\right)\left(i_{1}, i_{2}, \ldots, i_{n}\right)} \mathbf{x}(t)+\mathbf{B}_{\left(q_{1}, q_{2}, \ldots, q_{n}\right)\left(i_{1}, i_{2}, \ldots, i_{n}\right)} \mathbf{u}(t)\right\},
\end{aligned}
$$

where the normalized membership function of the $\operatorname{RMR}_{\left(q_{1}, q_{2}, \ldots, q_{n}\right)}$ is

$$
\eta_{\left(q_{1}, q_{2}, \ldots, q_{n}\right)}(\mathbf{x}(t))=\prod_{j=1}^{n} N_{\left(j, q_{j}\right)}\left(x_{j}(t)\right)
$$

and the fire strength of the $\operatorname{LMR}_{\left(i_{1}, i_{2}, \ldots, i_{n}\right)}$ in $R_{\left(q_{1}, q_{2}, \ldots, q_{n}\right)}$ is

$$
w_{\left(q_{1}, q_{2}, \ldots, q_{n}\right)\left(i_{1}, i_{2}, \ldots, i_{n}\right)}(\mathbf{x}(t))=\prod_{j=1}^{n} M_{q_{j},\left(j, i_{j}\right)}\left(x_{j}(t)\right)
$$

for all $t$. And the term $M_{q_{j},\left(j, i_{j}\right)}\left(x_{j}(t)\right)$ is the grade of membership of $x_{j}(t)$ in $M_{q_{j},\left(j, i_{j}\right)}$.

The normalized membership function of the $\operatorname{LMR}_{\left(i_{1}, i_{2}, \ldots, i_{n}\right)}$ in $R_{\left(q_{1}, q_{2}, \ldots, q_{n}\right)}$ can be described by

$$
\begin{aligned}
& h_{\left(q_{1}, q_{2}, \ldots, q_{n}\right)\left(i_{1}, i_{2}, \ldots, i_{n}\right)}(\mathbf{x}(t)) \\
& \quad=\frac{w_{\left(q_{1}, q_{2}, \ldots, q_{n}\right)\left(i_{1}, i_{2}, \ldots, i_{n}\right)}(\mathbf{x}(t))}{\sum_{i_{1}=1}^{r_{1 q_{1}}} \sum_{i_{2}=1}^{r_{2 q_{2}}} \cdots \sum_{i_{n}=1}^{r_{n q_{n}}} w_{\left(q_{1}, q_{2}, \ldots, q_{n}\right)\left(i_{1}, i_{2}, \ldots, i_{n}\right)}(\mathbf{x}(t))}
\end{aligned}
$$

with

$$
\begin{gathered}
\sum_{i_{1}=1}^{r_{1 q_{1}}} \sum_{i_{2}=1}^{r_{2 q_{2}}} \ldots \sum_{i_{n}=1}^{r_{n q_{n}}} h_{\left(q_{1}, q_{2}, \ldots, q_{n}\right)\left(i_{1}, i_{2}, \ldots, i_{n}\right)}(\mathbf{x}(t))=1, \\
h_{\left(q_{1}, q_{2}, \ldots, q_{n}\right)\left(i_{1}, i_{2}, \ldots, i_{n}\right)}(\mathbf{x}(t)) \geq 0, \\
i_{j}=1,2, \ldots, r_{j q_{j}}, \quad j=1,2, \ldots, n .
\end{gathered}
$$

Example 5. Consider a system with two state variables $x_{1}(t)$ and $x_{2}(t)$. The T-S fuzzy model is assumed to have 16 subsystems and four fuzzy sets for each state variable. A state space with two dimension can be divided into the following four regions: $R(0,0), R(0,1), R(1,0)$, and $R(1,1)$ as shown in Figure 5. The T-S fuzzy model that uses the process of reconstruction can obtain the following new T-S fuzzy model as shown in Figure 6. The new T-S fuzzy model has 25 subsystems and five fuzzy sets for each state variable

$$
\begin{aligned}
& A_{(0,1)}=M_{(1,2)}\left(x_{1}(t)\right) A_{(2,1)}+M_{(1,3)}\left(x_{1}(t)\right) A_{(3,1)}, \\
& A_{(0,2)}=M_{(1,2)}\left(x_{1}(t)\right) A_{(2,2)}+M_{(1,3)}\left(x_{1}(t)\right) A_{(3,2)}, \\
& A_{(0,3)}=M_{(1,2)}\left(x_{1}(t)\right) A_{(2,3)}+M_{(1,3)}\left(x_{1}(t)\right) A_{(3,3)}, \\
& A_{(0,4)}=M_{(1,2)}\left(x_{1}(t)\right) A_{(2,4)}+M_{(1,3)}\left(x_{1}(t)\right) A_{(3,4)}, \\
& A_{(1,0)}=M_{(2,2)}\left(x_{2}(t)\right) A_{(1,2)}+M_{(2,3)}\left(x_{2}(t)\right) A_{(1,3)}, \\
& A_{(2,0)}=M_{(2,2)}\left(x_{2}(t)\right) A_{(2,2)}+M_{(2,3)}\left(x_{2}(t)\right) A_{(2,3)},
\end{aligned}
$$

$$
\begin{aligned}
A_{(3,0)}= & M_{(2,2)}\left(x_{2}(t)\right) A_{(3,2)}+M_{(2,3)}\left(x_{2}(t)\right) A_{(3,3)}, \\
A_{(4,0)}= & M_{(2,2)}\left(x_{2}(t)\right) A_{(4,2)}+M_{(2,3)}\left(x_{2}(t)\right) A_{(4,3)}, \\
A_{(0,0)}= & M_{(1,2)}\left(x_{1}(t)\right) M_{(2,2)}\left(x_{2}(t)\right) A_{(2,2)} \\
& +M_{(1,3)}\left(x_{1}(t)\right) M_{(2,2)}\left(x_{2}(t)\right) A_{(3,2)} \\
& +M_{(1,2)}\left(x_{1}(t)\right) M_{(2,3)}\left(x_{2}(t)\right) A_{(2,3)} \\
& +M_{(1,3)}\left(x_{1}(t)\right) M_{(2,3)}\left(x_{2}(t)\right) A_{(3,3)} .
\end{aligned}
$$

For the new T-S fuzzy model in Figure 6, it can be separated at region boundaries and depicted as Figure 7.

\subsection{Relaxed Stability Conditions Based on Switching Quad-} ratic Lyapunov Function. Consider

Region Function Rule $\left(q_{1}, q_{2}, \ldots, q_{n}\right)-\left(\operatorname{RFR}_{\left(q_{1}, q_{2}, \ldots, q_{n}\right)}\right)$ :

IF $x_{1}(t)$ is $N_{\left(1, q_{1}\right)}$ and $x_{2}(t)$ is $N_{\left(2, q_{2}\right)} \cdots$ and $x_{n}(t)$ is $N_{\left(n, q_{n}\right)}$,

$\operatorname{THEN} V(\mathbf{x}(t))=\mathbf{x}^{T}(t) \mathbf{P}_{\left(q_{1}, q_{2}, \ldots, q_{n}\right)} \mathbf{x}(t)$,

$$
q_{j}=0,1, \quad j=1,2, \ldots, n,
$$

where $\operatorname{RFR}_{\left(q_{1}, q_{2}, \ldots, q_{n}\right)}$ denotes the region function rule $\left(q_{1}, q_{2}, \ldots, q_{n}\right) . \mathbf{P}_{\left(q_{1}, q_{2}, \ldots, q_{n}\right)} \in \mathfrak{R}^{n \times n}$ is a set of positive-definite symmetric matrices.

Based on the CAD for the $\operatorname{RFR}_{\left(q_{1}, q_{2}, \ldots, q_{n}\right)}$. The overall SFQLF is given as

$$
\begin{aligned}
& V(\mathbf{x}(t)) \\
& =\sum_{q_{1}=0}^{1} \sum_{q_{2}=0}^{1} \ldots \sum_{q_{n}=0}^{1} \eta_{\left(q_{1}, q_{2}, \ldots, q_{n}\right)}(\mathbf{x}(t)) \mathbf{x}^{T}(t) \mathbf{P}_{\left(q_{1}, q_{2}, \ldots, q_{n}\right)} \mathbf{x}(t) .
\end{aligned}
$$

This function is continuous on region boundaries if $\mathbf{P}_{\left(q_{1}, q_{2}, \ldots, q_{n}\right)}$ satisfies the following constraint:

$$
\mathbf{P}_{\left(q_{1}, q_{2}, \ldots, q_{n}\right)}=\left[\begin{array}{cccc}
p_{11 q_{1}} & p_{12\left(2 q_{1}+q_{2}\right)} & \cdots & p_{1 n\left(2 q_{1}+q_{n}\right)} \\
p_{12\left(2 q_{1}+q_{2}\right)} & p_{22 q_{2}} & \cdots & p_{2 n\left(2 q_{2}+q_{n}\right)} \\
\vdots & \vdots & \ddots & \vdots \\
p_{1 n\left(2 q_{1}+q_{n}\right)} & p_{2 n\left(2 q_{2}+q_{n}\right)} & \cdots & p_{n n q_{n}}
\end{array}\right] .
$$

The autonomous switching T-S fuzzy system via vertex expression with (22) can be represented as

$$
\begin{aligned}
\dot{\mathbf{x}}(t)= & \sum_{q_{1}=0}^{1} \sum_{q_{2}=0}^{1} \ldots \sum_{q_{n}=0}^{1} \sum_{i_{1}=1}^{r_{1 q_{1}}} \sum_{i_{2}=1}^{r_{2 q_{2}}} \ldots \sum_{i_{n}=1}^{r_{n q_{n}}} \eta_{\left(q_{1}, q_{2}, \ldots, q_{n}\right)}(\mathbf{x}(t)) \\
& \times h_{\left(q_{1}, q_{2}, \ldots, q_{n}\right)\left(i_{1}, i_{2}, \ldots, i_{n}\right)}(\mathbf{x}(t)) \mathbf{A}_{\left(q_{1}, q_{2}, \ldots, q_{n}\right)\left(i_{1}, i_{2}, \ldots, i_{n}\right)} \mathbf{x}(t) .
\end{aligned}
$$




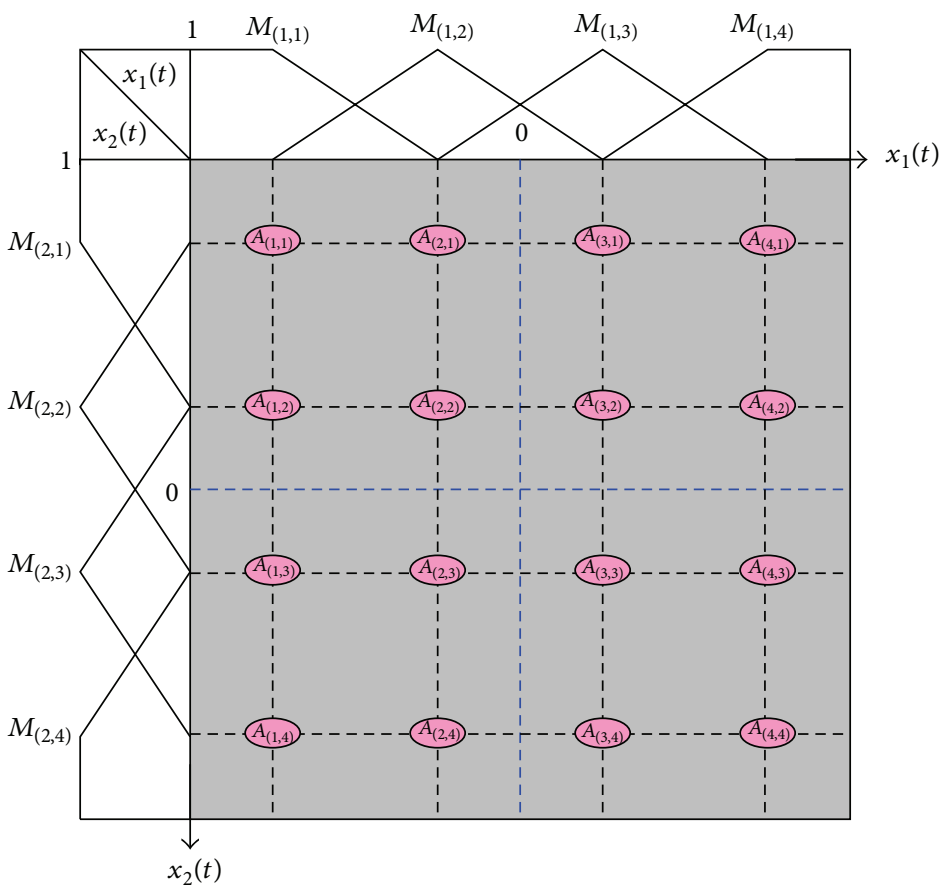

FIGURE 5: Rules illustration of Example 5.

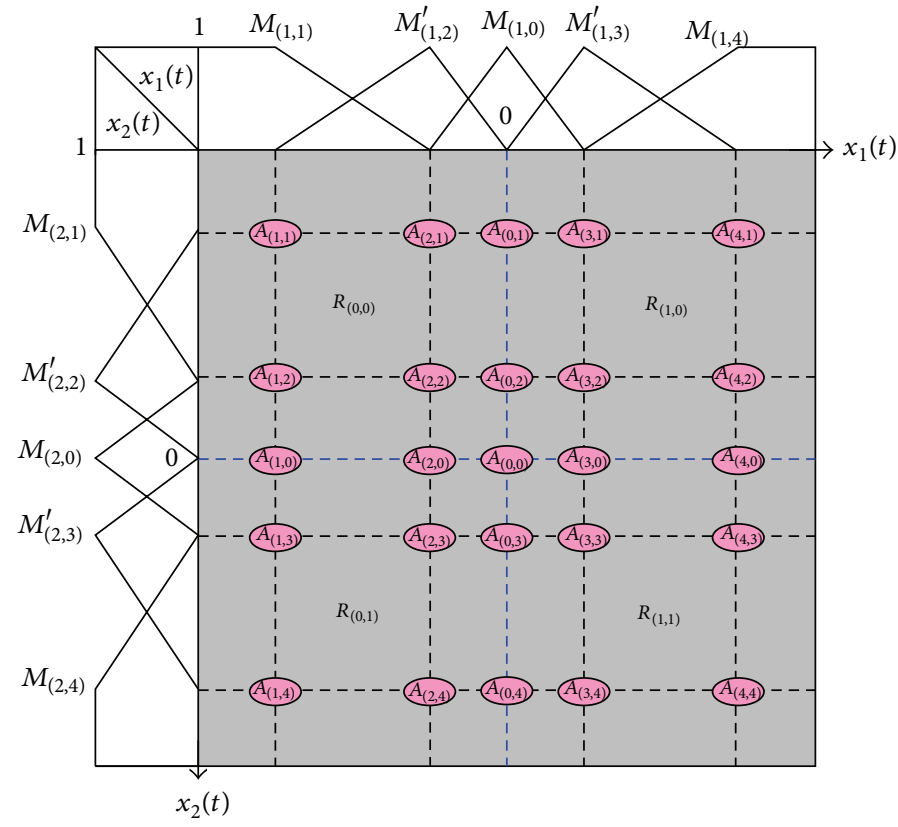

FIGURE 6: Rules illustration of Example 5 by the first process of reconstruction. 

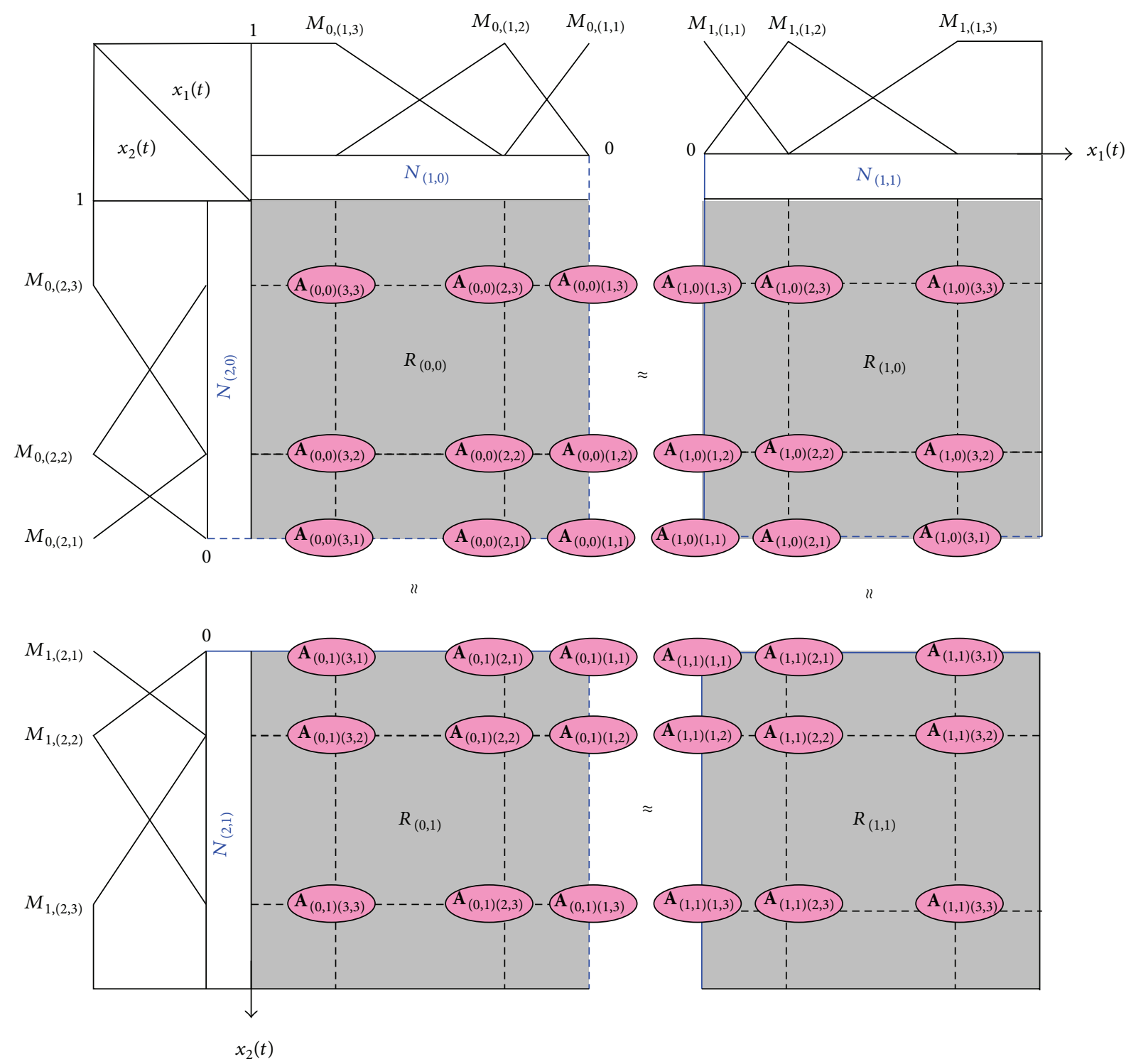

FIGURE 7: Rules illustration of Example 5 by the second process of reconstruction.

By reconstruction of the switching T-S fuzzy model, we can obtain relaxed stability conditions for autonomous switching T-S fuzzy systems via vertex expression (31) based on SFQLF.

Theorem 6. The equilibrium of the reconstructive autonomous switching T-S fuzzy system (31) is globally asymptotically stable if there exist a set of matrices $\mathbf{P}_{\left(q_{1}, q_{2}, \ldots, q_{n}\right)}$ such that (30) and (32):

$$
\begin{aligned}
& \mathbf{P}_{\left(q_{1}, q_{2}, \ldots, q_{n}\right)}>\mathbf{0}, \quad q_{j}=0,1, \quad j=1,2, \ldots, n, \\
& \mathbf{A}_{\left(q_{1}, q_{2}, \ldots, q_{n}\right)\left(i_{1}, i_{2}, \ldots, i_{n}\right)}^{T} \mathbf{P}_{\left(q_{1}, q_{2}, \ldots, q_{n}\right)} \\
& \quad+\mathbf{P}_{\left(q_{1}, q_{2}, \ldots, q_{n}\right)} \mathbf{A}_{\left(q_{1}, q_{2}, \ldots, q_{n}\right)\left(i_{1}, i_{2}, \ldots, i_{n}\right)}<\mathbf{0}
\end{aligned}
$$

$$
q_{j}=0,1, \quad i_{j}=1,2, \ldots, r_{j q_{j}}, \quad j=1,2, \ldots, n .
$$

Proof. Consider a candidate of Lyapunov function (29):

$$
\begin{aligned}
\dot{V}(\mathbf{x}(t))= & \sum_{q_{1}=0}^{1} \sum_{q_{2}=0}^{1} \cdots \sum_{q_{n}=0}^{1} \eta_{\left(q_{1}, q_{2}, \ldots, q_{n}\right)}(\mathbf{x}(t)) \\
& \times\left\{\dot{\mathbf{x}}^{T}(t) \mathbf{P}_{\left(q_{1}, q_{2}, \ldots, q_{n}\right)} \mathbf{x}(t)+\mathbf{x}^{T}(t) \mathbf{P}_{\left(q_{1}, q_{2}, \ldots, q_{n}\right)} \dot{\mathbf{x}}(t)\right\} .
\end{aligned}
$$

By (22), we manipulate the above equation and it then yields (34) (see below). Thus, from (30) and (32), the considered TS fuzzy system (31) is asserted to be globally asymptotically stable. 


$$
\begin{aligned}
\dot{V}(\mathbf{x}(t))= & \sum_{q_{1}=0}^{1} \sum_{q_{2}=0}^{1} \ldots \sum_{q_{n}=0}^{1} \eta_{\left(q_{1}, q_{2}, \ldots, q_{n}\right)}(\mathbf{x}(t)) \\
& \times\left\{\left(\sum_{i_{1}=1 i_{2}=1}^{r_{1 q_{1}}} \sum_{2 q_{2}}^{r_{2}} \ldots \sum_{i_{n}=1}^{r_{n q_{n}}} h_{\left(q_{1}, q_{2}, \ldots, q_{n}\right)\left(i_{1}, i_{2}, \ldots, i_{n}\right)}(\mathbf{x}(t)) \mathbf{x}^{T}(t) \mathbf{A}_{\left(q_{1}, q_{2}, \ldots, q_{n}\right)\left(i_{1}, i_{2}, \ldots, i_{n}\right)}^{T}\right) \times \mathbf{P}_{\left(q_{1}, q_{2}, \ldots, q_{n}\right)} \mathbf{x}(t)\right. \\
& \left.+\mathbf{x}^{T}(t) \mathbf{P}_{\left(q_{1}, q_{2}, \ldots, q_{n}\right)} \times\left(\sum_{i_{1}=1 i_{2}=1}^{r_{1 q_{1}} r_{2 q_{2}}} \ldots \sum_{i_{n}=1}^{r_{n q_{n}}} h_{\left(q_{1}, q_{2}, \ldots, q_{n}\right)\left(i_{1}, i_{2}, \ldots, i_{n}\right)}(\mathbf{x}(t)) \mathbf{A}_{\left(q_{1}, q_{2}, \ldots, q_{n}\right)\left(i_{1}, i_{2}, \ldots, i_{n}\right)} \mathbf{x}(t)\right)\right\} \\
= & \sum_{q_{1}=0}^{1} \sum_{q_{2}=0}^{1} \ldots \sum_{q_{n}=0}^{1} \sum_{i_{1}=1}^{r_{1 q_{1}}} \sum_{i_{2}=1}^{r_{2 q_{2}}} \ldots \sum_{i_{n}=1}^{r_{n q_{n}}} \eta_{\left(q_{1}, q_{2}, \ldots, q_{n}\right)}(\mathbf{x}(t)) h_{\left(q_{1}, q_{2}, \ldots, q_{n}\right)\left(i_{1}, i_{2}, \ldots, i_{n}\right)}(\mathbf{x}(t)) \\
& \times \mathbf{x}^{T}(t)\left\{\mathbf{A}_{\left(q_{1}, q_{2}, \ldots, q_{n}\right)\left(i_{1}, i_{2}, \ldots, i_{n}\right)}^{T} \mathbf{P}_{\left(q_{1}, q_{2}, \ldots, q_{n}\right)} \mathbf{x}(t)+\mathbf{P}_{\left(q_{1}, q_{2}, \ldots, q_{n}\right)} \mathbf{A}_{\left(q_{1}, q_{2}, \ldots, q_{n}\right)\left(i_{1}, i_{2}, \ldots, i_{n}\right)}\right\} \mathbf{x}(t) .
\end{aligned}
$$

\section{Illustrative Example}

Revisit Example 1 in the previous work [26]. By our proposed approach, we can beforehand use the reconstruction procedure for this T-S fuzzy model and thus can suitably perform the stability verification by Theorem 6 with SFQLF described in the sequel.

Example 7. Consider the following reconstructed switching T-S fuzzy model:

$\operatorname{RMR}_{(0,0)}: \operatorname{IF} x_{1}(t)$ is $N_{(1,0)}$ and $x_{2}(t)$ is $N_{(2,0)}$

THEN $\operatorname{LMR}_{(1,1)}$ : IF $x_{1}(t)$ is $M_{0,(1,1)}$ and $x_{2}(t)$ is $M_{0,(2,1)}$

$$
\operatorname{THEN} \dot{\mathbf{x}}(t)=\mathbf{A}_{(0,0)(1,1)} \mathbf{x}(t)
$$

$\operatorname{LMR}_{(2,1)}:$ IF $x_{1}(t)$ is $M_{0,(1,2)}$ and $x_{2}(t)$ is $M_{0,(2,1)}$

$$
\operatorname{THEN} \dot{\mathbf{x}}(t)=\mathbf{A}_{(0,0)(2,1)} \mathbf{x}(t)
$$

$\operatorname{LMR}_{(1,2)}$ : IF $x_{1}(t)$ is $M_{0,(1,1)}$ and $x_{2}(t)$ is $M_{0,(2,2)}$

$$
\operatorname{THEN} \dot{\mathbf{x}}(t)=\mathbf{A}_{(0,0)(1,2)} \mathbf{x}(t)
$$

$\operatorname{LMR}_{(2,2)}$ : IF $x_{1}(t)$ is $M_{0,(1,2)}$ and $x_{2}(t)$ is $M_{0,(2,2)}$

$$
\text { THEN } \dot{\mathbf{x}}(t)=\mathbf{A}_{(0,0)(2,2)} \mathbf{x}(t)
$$

$\operatorname{RMR}_{(1,0)}$ : IF $x_{1}(t)$ is $N_{(1,1)}$ and $x_{2}(t)$ is $N_{(2,0)}$

THEN $\operatorname{LMR}_{(1,1)}$ : IF $x_{1}(t)$ is $M_{1,(1,1)}$ and $x_{2}(t)$ is $M_{0,(2,1)}$

$$
\operatorname{THEN} \dot{\mathbf{x}}(t)=\mathbf{A}_{(1,0)(1,1)} \mathbf{x}(t)
$$

$\operatorname{LMR}_{(2,1)}$ : IF $x_{1}(t)$ is $M_{1,(1,2)}$ and $x_{2}(t)$ is $M_{0,(2,1)}$

$$
\operatorname{THEN} \dot{\mathbf{x}}(t)=\mathbf{A}_{(1,0)(2,1)} \mathbf{x}(t)
$$

$\operatorname{LMR}_{(1,2)}:$ IF $x_{1}(t)$ is $M_{1,(1,1)}$ and $x_{2}(t)$ is $M_{0,(2,2)}$

$$
\operatorname{THEN} \dot{\mathbf{x}}(t)=\mathbf{A}_{(1,0)(1,2)} \mathbf{x}(t)
$$

$\operatorname{LMR}_{(2,2)}:$ IF $x_{1}(t)$ is $M_{1,(1,2)}$ and $x_{2}(t)$ is $M_{0,(2,2)}$

$$
\operatorname{THEN} \dot{\mathbf{x}}(t)=\mathbf{A}_{(1,0)(2,2)} \mathbf{x}(t)
$$

$\operatorname{RMR}_{(0,1)}:$ IF $x_{1}(t)$ is $N_{(1,0)}$ and $x_{2}(t)$ is $N_{(2,1)}$

THEN $\operatorname{LMR}_{(1,1)}$ : IF $x_{1}(t)$ is $M_{0,(1,1)}$ and $x_{2}(t)$ is $M_{1,(2,1)}$

$$
\operatorname{THEN} \dot{\mathbf{x}}(t)=\mathbf{A}_{(0,1)(1,1)} \mathbf{x}(t)
$$

$\operatorname{LMR}_{(2,1)}:$ IF $x_{1}(t)$ is $M_{0,(1,2)}$ and $x_{2}(t)$ is $M_{1,(2,1)}$

$$
\operatorname{THEN} \dot{\mathbf{x}}(t)=\mathbf{A}_{(0,1)(2,1)} \mathbf{x}(t)
$$

$\operatorname{LMR}_{(1,2)}:$ IF $x_{1}(t)$ is $M_{0,(1,1)}$ and $x_{2}(t)$ is $M_{1,(2,2)}$

$$
\operatorname{THEN~} \dot{\mathbf{x}}(t)=\mathbf{A}_{(0,1)(1,2)} \mathbf{x}(t)
$$

$\operatorname{LMR}_{(2,2)}:$ IF $x_{1}(t)$ is $M_{0,(1,2)}$ and $x_{2}(t)$ is $M_{1,(2,2)}$

$$
\operatorname{THEN} \dot{\mathbf{x}}(t)=\mathbf{A}_{(0,1)(2,2)} \mathbf{x}(t)
$$

$\operatorname{RMR}_{(1,1)}:$ IF $x_{1}(t)$ is $N_{(1,1)}$ and $x_{2}(t)$ is $N_{(2,1)}$

THEN $\operatorname{LMR}_{(1,1)}$ : IF $x_{1}(t)$ is $M_{1,(1,1)}$ and $x_{2}(t)$ is $M_{1,(2,1)}$

$$
\operatorname{THEN} \dot{\mathbf{x}}(t)=\mathbf{A}_{(1,1)(1,1)} \mathbf{x}(t)
$$

$\operatorname{LMR}_{(2,1)}:$ IF $x_{1}(t)$ is $M_{1,(1,2)}$ and $x_{2}(t)$ is $M_{1,(2,1)}$

$$
\operatorname{THEN} \dot{\mathbf{x}}(t)=\mathbf{A}_{(1,1)(2,1)} \mathbf{x}(t)
$$

$\operatorname{LMR}_{(1,2)}:$ IF $x_{1}(t)$ is $M_{1,(1,1)}$ and $x_{2}(t)$ is $M_{1,(2,2)}$

$$
\operatorname{THEN~} \dot{\mathbf{x}}(t)=\mathbf{A}_{(1,1)(1,2)} \mathbf{x}(t)
$$

$\operatorname{LMR}_{(2,2)}:$ IF $x_{1}(t)$ is $M_{1,(1,2)}$ and $x_{2}(t)$ is $M_{1,(2,2)}$

$$
\operatorname{THEN} \dot{\mathbf{x}}(t)=\mathbf{A}_{(1,1)(2,2)} \mathbf{x}(t),
$$



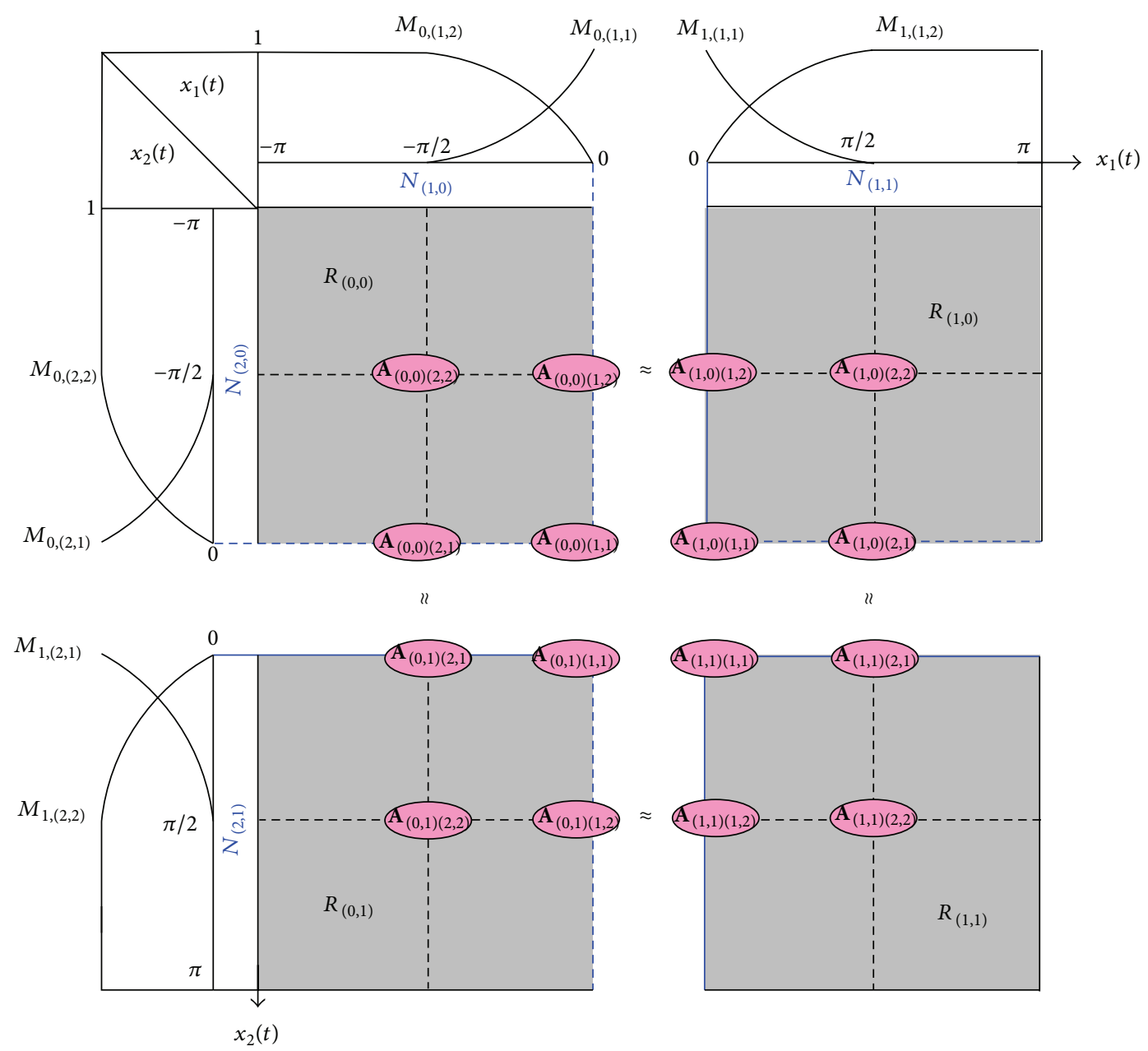

FIGURE 8: Rules illustration of switching T-S fuzzy model via vertex expression for Example 7.

where the original system matrices of the subsystem are

$$
\begin{gathered}
\mathbf{A}_{1}=\left[\begin{array}{cc}
-5 & -4 \\
-1 & a
\end{array}\right], \quad \mathbf{A}_{2}=\left[\begin{array}{cc}
-4 & -4 \\
\frac{1}{5}(3 b-2) & \frac{1}{5}(3 a-4)
\end{array}\right], \\
\mathbf{A}_{3}=\left[\begin{array}{cc}
-3 & -4 \\
\frac{1}{5}(2 b-3) & \frac{1}{5}(2 a-6)
\end{array}\right], \quad \mathbf{A}_{4}=\left[\begin{array}{cc}
-2 & -4 \\
b & -2
\end{array}\right] .
\end{gathered}
$$

By the reconstructing procedure, the considered system associated with rules illustration via vertex expression is depicted in Figure 8. Thus, the systems' matrices can be, respectively, calculated as

$$
\begin{gathered}
\mathbf{A}_{(0,0)(2,2)}=\mathbf{A}_{2}, \quad \mathbf{A}_{(0,0)(1,2)}=\frac{\mathbf{A}_{2}+\mathbf{A}_{4}}{2}, \\
\mathbf{A}_{(1,0)(1,2)}=\frac{\mathbf{A}_{2}+\mathbf{A}_{4}}{2}, \quad \mathbf{A}_{(1,0)(2,2)}=\mathbf{A}_{4}, \\
\mathbf{A}_{(0,0)(2,1)}=\frac{\mathbf{A}_{1}+\mathbf{A}_{2}}{2}, \quad \mathbf{A}_{(0,0)(1,1)}=\frac{\mathbf{A}_{1}+\mathbf{A}_{2}+\mathbf{A}_{3}+\mathbf{A}_{4}}{4},
\end{gathered}
$$

$$
\begin{gathered}
\mathbf{A}_{(1,0)(1,1)} \frac{\mathbf{A}_{1}+\mathbf{A}_{2}+\mathbf{A}_{3}+\mathbf{A}_{4}}{4}, \quad \mathbf{A}_{(1,0)(2,1)}=\frac{\mathbf{A}_{3}+\mathbf{A}_{4}}{2}, \\
\mathbf{A}_{(0,1)(2,1)}=\frac{\mathbf{A}_{1}+\mathbf{A}_{2}}{2}, \quad \mathbf{A}_{(0,1)(1,1)} \frac{\mathbf{A}_{1}+\mathbf{A}_{2}+\mathbf{A}_{3}+\mathbf{A}_{4}}{4}, \\
\mathbf{A}_{(1,1)(1,1)}=\frac{\mathbf{A}_{1}+\mathbf{A}_{2}+\mathbf{A}_{3}+\mathbf{A}_{4}}{4}, \quad \mathbf{A}_{(1,1)(2,1)}=\frac{\mathbf{A}_{3}+\mathbf{A}_{4}}{2}, \\
\mathbf{A}_{(0,0)(2,2)}=\mathbf{A}_{1}, \quad \mathbf{A}_{(0,1)(1,2)}=\frac{\mathbf{A}_{1}+\mathbf{A}_{3}}{2}, \\
\mathbf{A}_{(1,1)(1,2)}=\frac{\mathbf{A}_{1}+\mathbf{A}_{3}}{2}, \quad \mathbf{A}_{(1,1)(2,2)}=\mathbf{A}_{3},
\end{gathered}
$$

with

$$
\begin{aligned}
& N_{(1,0)}\left(x_{1}(t)\right)= \begin{cases}1, & \text { for } x_{1}(t)<0 \\
0, & \text { for } x_{1}(t) \geq 0,\end{cases} \\
& N_{(1,1)}\left(x_{1}(t)\right)= \begin{cases}1, & \text { for } x_{1}(t) \geq 0 \\
0, & \text { for } x_{1}(t)<0,\end{cases}
\end{aligned}
$$




$$
\begin{aligned}
& N_{(2,0)}\left(x_{2}(t)\right)= \begin{cases}1, & \text { for } x_{2}(t)<0 \\
0, & \text { for } x_{2}(t) \geq 0\end{cases} \\
& N_{(2,1)}\left(x_{2}(t)\right)= \begin{cases}1, & \text { for } x_{2}(t) \geq 0 \\
0, & \text { for } x_{2}(t)<0 .\end{cases} \\
& M_{0,(1,2)}\left(x_{1}(t)\right)= \begin{cases}1, & \text { for } x_{1}(t) \leq-\frac{\pi}{2} \\
-\sin \left(x_{1}(t)\right), & \text { for }-\frac{\pi}{2} \leq x_{1}(t) \leq 0,\end{cases} \\
& M_{0,(1,1)}\left(x_{1}(t)\right)= \begin{cases}0, & \text { for } x_{1}(t) \leq-\frac{\pi}{2} \\
1+\sin \left(x_{1}(t)\right), & \text { for }-\frac{\pi}{2} \leq x_{1}(t) \leq 0\end{cases} \\
& M_{1,(1,1)}\left(x_{1}(t)\right)= \begin{cases}1-\sin \left(x_{1}(t)\right), & \text { for } x_{1}(t) \leq \frac{\pi}{2} \\
0, & \text { for } \frac{\pi}{2} \leq x_{1}(t)\end{cases} \\
& M_{1,(1,2)}\left(x_{1}(t)\right)= \begin{cases}\sin \left(x_{1}(t)\right) & \text { for } 0 \leq x_{1}(t) \leq \frac{\pi}{2} \\
1 & \text { for } \frac{\pi}{2} \leq x_{1}(t)\end{cases} \\
& M_{0,(2,2)}\left(x_{2}(t)\right)= \begin{cases}1, & \text { for } x_{2}(t) \leq-\frac{\pi}{2} \\
-\sin \left(x_{2}(t)\right), & \text { for }-\frac{\pi}{2} \leq x_{2}(t) \leq 0\end{cases} \\
& M_{0,(2,1)}\left(x_{2}(t)\right)= \begin{cases}0, & \text { for } x_{2}(t) \leq-\frac{\pi}{2} \\
1+\sin \left(x_{2}(t)\right), & \text { for }-\frac{\pi}{2} \leq x_{2}(t) \leq 0\end{cases} \\
& M_{1,(2,1)}\left(x_{1}(t)\right)= \begin{cases}1-\sin \left(x_{2}(t)\right), & \text { for } x_{2}(t) \leq \frac{\pi}{2} \\
0, & \text { for } \frac{\pi}{2} \leq x_{2}(t)\end{cases} \\
& M_{1,(2,2)}\left(x_{2}(t)\right)= \begin{cases}\sin \left(x_{2}(t)\right) & \text { for } 0 \leq x_{2}(t) \leq \frac{\pi}{2} \\
1 & \text { for } \frac{\pi}{2} \leq x_{2}(t)\end{cases}
\end{aligned}
$$

In this example, by applying a current tool, Matlab software, we experimentally attain a widely feasible region for the parameters' pair $(a, b)$ via SQLF method compared to that of the CQLF method. The feasible parameter pairs $(a, b)$ of the CQLF and the SQLF methods are shown in Figures 9 and 10 , respectively. By observation, the proposed approach can dramatically lessen the conservatism of the stability assurance.

\section{Conflict of Interests}

The authors declare that there is no conflict of interests regarding the publication of this paper.

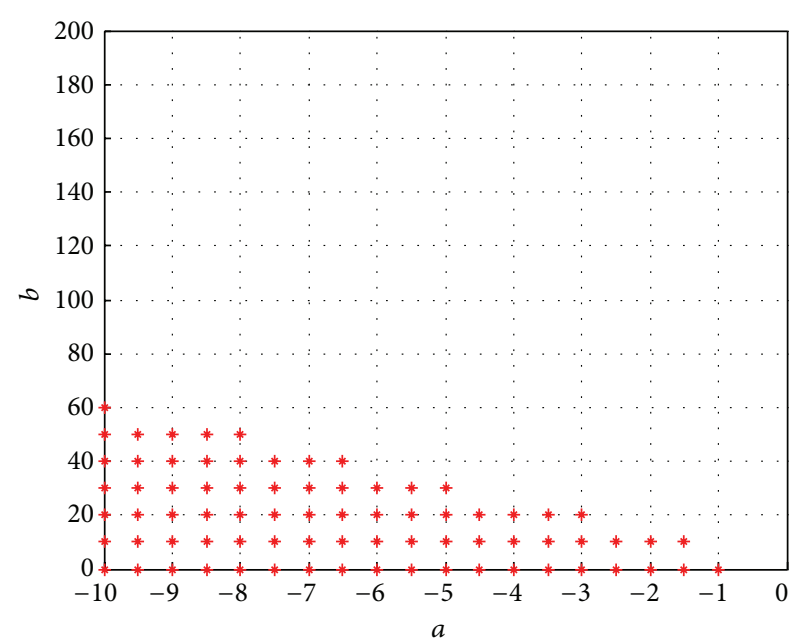

FIgURE 9: Feasible area for Example 7 that uses the CQLF method.

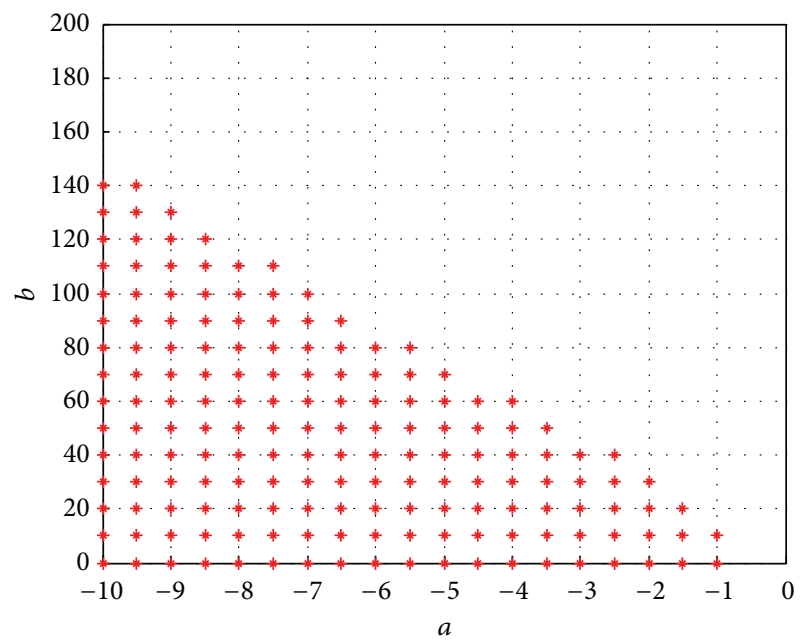

FIGURE 10: Feasible area for Example 7 that uses the SQLF method.

\section{References}

[1] L. A. Zadeh, "Outline of new approach to the analysis of complex systems and decision processes," IEEE Transactions on Systems, Man, and Cybernetics, vol. 3, pp. 28-44, 1973.

[2] T. Takagi and M. Sugeno, "Fuzzy identification of systems and its applications tomodeling and control," IEEE Transactions on Systems, Man, and Cybernetics Part B, vol. SMC-15, no. 2, pp. 116-132, 1985.

[3] K. Tanaka and M. Sugeno, "Stability analysis and design of fuzzy control systems," Fuzzy Sets and Systems, vol. 45, no. 2, pp. 135$156,1992$.

[4] K. Tanaka and M. Sano, "A robust stabilization problem of fuzzy control systems and its application to backing up control of a truck-trailer," IEEE Transactions on Fuzzy Systems, vol. 2, no. 2, pp. 119-134, 1994.

[5] P. P. Angelov and D. P. Filev, "An approach to online identification of Takagi-Sugeno fuzzy models," IEEE Transactions on Systems, Man, and Cybernetics, Part B: Cybernetics, vol. 34, no. 1, pp. 484-498, 2004. 
[6] I. Kar, P. K. Patchaikani, and L. Behera, "On balancing a cartpole system using T-S fuzzy model," Fuzzy Sets and Systems, vol. 207, pp. 94-110, 2012.

[7] J. X. Xu, Z. Q. Guo, and T. H. Lee, "Design and implementation of a Takagi-Sugeno-type fuzzy logic controller on a two-wheeled mobile robot," IEEE Transactions on Industrial Electronics, vol. 60, no. 12, pp. 5717-5727, 2013.

[8] L. Zhang, X. Chang, and H. R. Karimi, "Fuzzy modeling and control for a class of inverted pendulum system," Abstract and Applied Analysis, vol. 2014, Article ID 936868, 6 pages, 2014.

[9] H. O. Wang, K. Tanaka, and M. F. Griffin, "Parallel distributed compensation of nonlinear systems by Takagi-Sugeno fuzzy model," in Proceedings of 4th IEEE International Conference on Fuzzy Systems and the 2nd International Fuzzy Engineering Symposium, vol. 2, pp. 531-538, Yokohama, Japan, March 1995.

[10] H. O. Wang, K. Tanaka, and M. F. Griffin, "An approach to fuzzy control of nonlinear systems: stability and design issues," IEEE Transactions on Fuzzy Systems, vol. 4, no. 1, pp. 14-23, 1996.

[11] K. Tanaka, T. Ikeda, and H. O. Wang, "Robust stabilization of a class of uncertain nonlinear system via fuzzy control," IEEE Transactions on Fuzzy Systems, vol. 4, pp. 1-13, 1996.

[12] K. Tanaka, T. Ikeda, and H. O. Wang, "Fuzzy regulators and fuzzy observers: relaxed stability conditions and LMI-based designs," IEEE Transactions on Fuzzy Systems, vol. 6, no. 2, pp. 250-265, 1998.

[13] M. Narimani, H. K. Lam, R. Dilmaghani, and C. Wolfe, "LMIbased stability analysis of fuzzy-model-based control systems using approximated polynomial membership functions," IEEE Transactions on Systems, Man, and Cybernetics, Part B: Cybernetics, vol. 41, no. 3, pp. 713-724, 2011.

[14] L. K. Wong, F. H. F. Leung, and P. K. S. Tam, "Lyapunov function-based design of fuzzy logic controllers and its application on combining controllers," IEEE Transactions on Industrial Electronics, vol. 45, no. 3, pp. 502-509, 1998.

[15] M. Sugeno, "On stability of fuzzy systems expressed by fuzzy rules with singleton consequents," IEEE Transactions on Fuzzy Systems, vol. 7, pp. 201-224, 1999.

[16] E. Kim and H. Lee, "New approaches to relaxed quadratic stability condition of fuzzy control systems," IEEE Transactions on Fuzzy Systems, vol. 8, pp. 523-533, 2000.

[17] K. Tanaka and H. O. Wang, Fuzzy Control Systems Design and Analysis: A Linear Matrix Inequality Approach, John Wiley \& Sons, New York, NY, USA, 2001.

[18] H. D. Tuan, P. Apkarian, T. Narikiyo, and Y. Yamamoto, "Parameterized linear matrix inequality techniques in fuzzy control system design," IEEE Transactions on Fuzzy Systems, vol. 9, no. 2, pp. 324-332, 2001.

[19] J.-M. Zhang, R.-H. Li, and P.-A. Zhang, "Stability analysis and systematic design of fuzzy control systems," Fuzzy Sets and Systems, vol. 120, no. 1, pp. 65-72, 2001.

[20] M. Johansson, A. Rantzer, and K. E. Årzén, "Piecewise quadratic stability of fuzzy systems," IEEE Transactions on Fuzzy Systems, vol. 7, no. 6, pp. 713-722, 1999.

[21] M. Feng and C. J. Harris, "Piecewise Lyapunov stability conditions of fuzzy systems," IEEE Transactions on Systems, Man, and Cybernetics, Part B, vol. 31, no. 2, pp. 259-262, 2001.

[22] Z. H. Xiu and G. Ren, "Stability analysis and systematic design of Takagi-Sugeno fuzzy control systems," Fuzzy Sets and Systems, vol. 151, no. 1, pp. 119-138, 2005.

[23] H. Ohtake, K. Tanaka, and H. O. Wang, "Switching fuzzy controller design based on switching Lyapunov function for a class of nonlinear systems," IEEE Transactions on Systems, Man, and Cybernetics B, vol. 36, no. 1, pp. 13-23, 2006.

[24] W. J. Wang, Y. J. Chen, and C. H. Sun, "Relaxed stabilization criteria for discrete-time T-S fuzzy control systems based on a switching fuzzy model and piecewise Lyapunov function," IEEE Transactions on Fuzzy Systems, vol. 37, no. 3, pp. 551-559, 2007.

[25] P. Gahinet, A. Nemirovski, A. Laub, and M. Chilali, LMI Control Toolbox, The MathWorks Inc., 1995.

[26] B.-J. Rhee and S. Won, "A new fuzzy Lyapunov function approach for a Takagi-Sugeno fuzzy control system design," Fuzzy Sets and Systems, vol. 157, no. 9, pp. 1211-1228, 2006. 


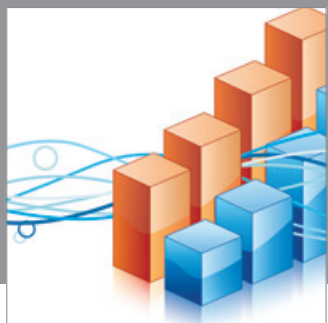

Advances in

Operations Research

mansans

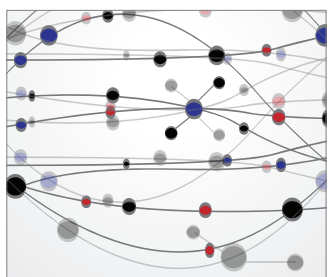

The Scientific World Journal
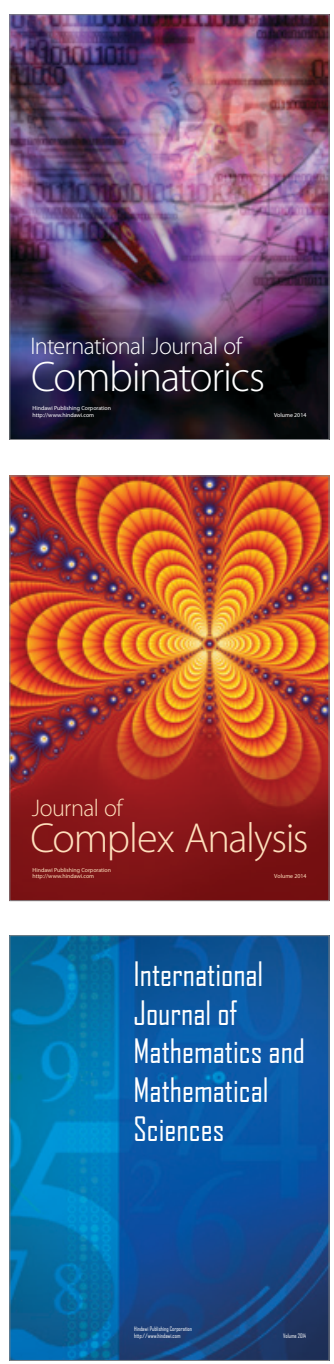
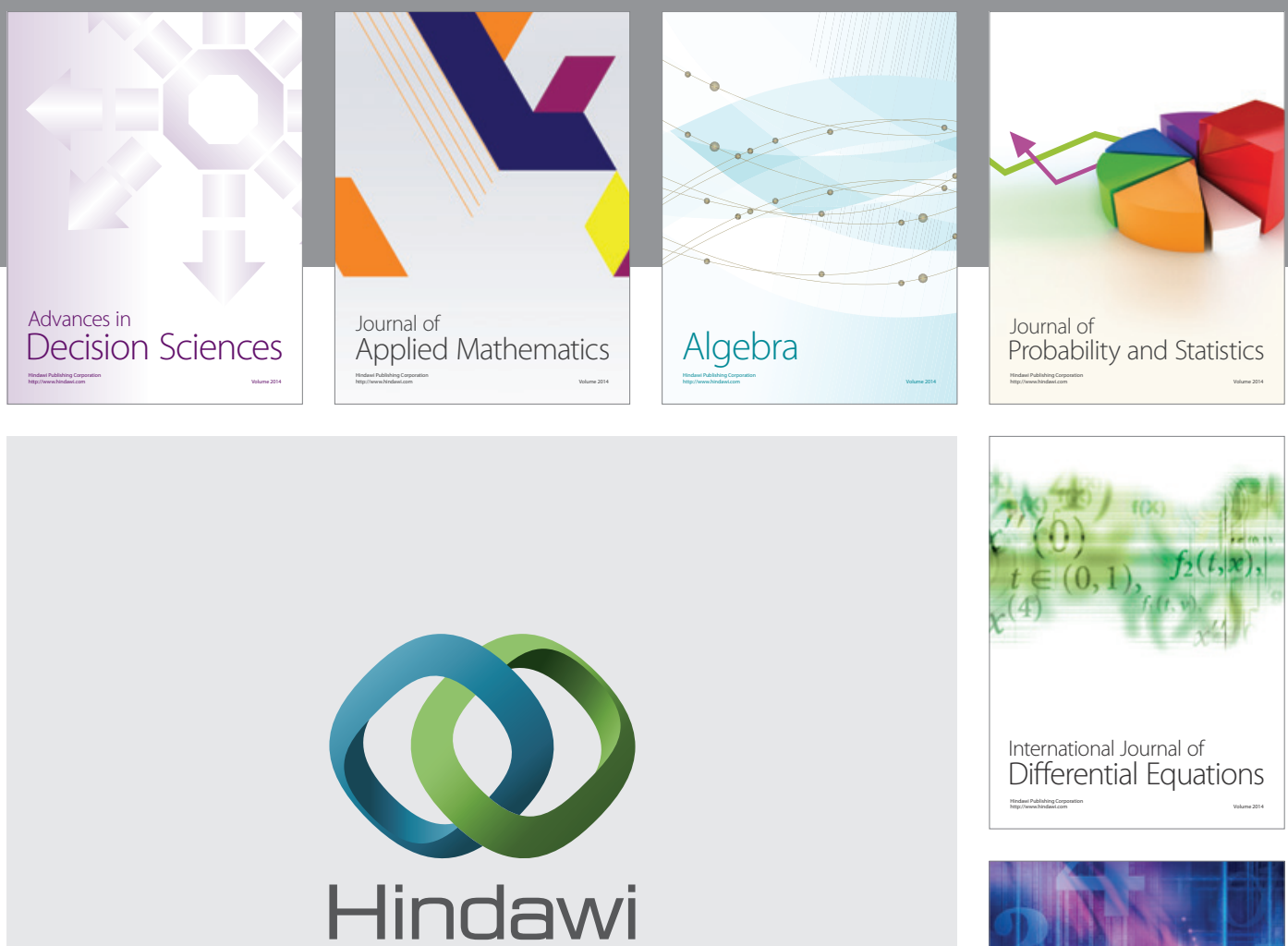

Submit your manuscripts at http://www.hindawi.com
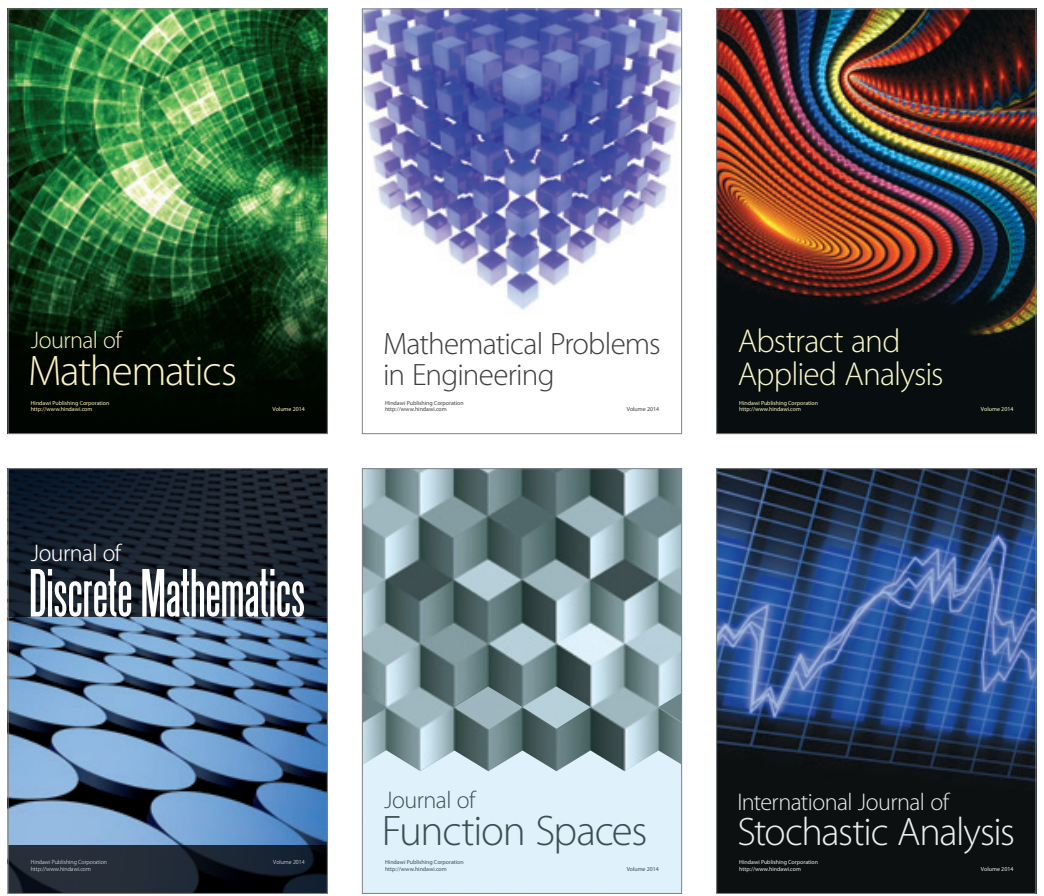

Journal of

Function Spaces

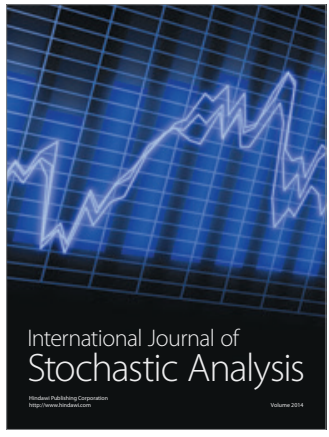

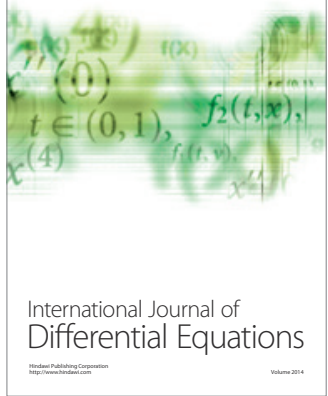
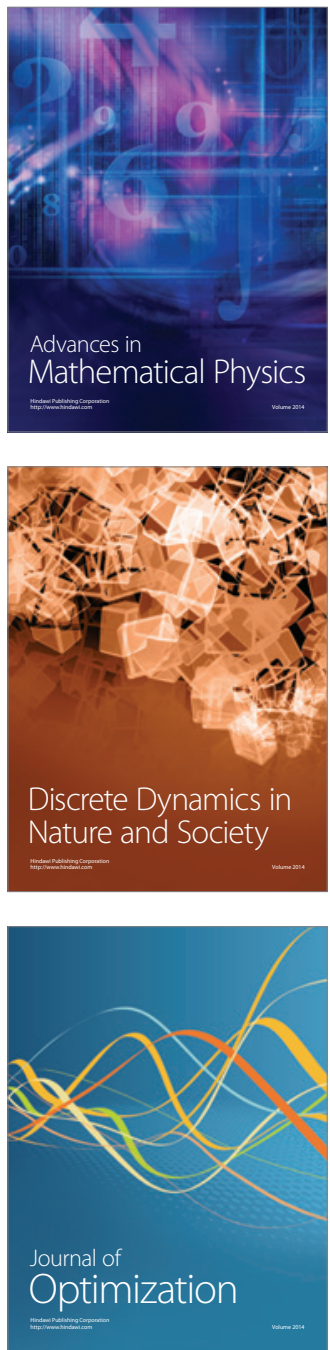